\title{
Learning in Distributed Low-Stakes Teams
}

\author{
Stephen MacNeil \\ Software and Information \\ Systems \\ UNC at Charlotte \\ smacnei2@uncc.edu
}

\author{
Celine Latulipe \\ Software and Information \\ Systems \\ UNC at Charlotte \\ clatulip@uncc.edu
}

\author{
Aman Yadav \\ Educational Psychology and \\ Educational Technology \\ Michigan State University \\ ayadav@msu.edu
}

\begin{abstract}
Active learning is important in computer science education, where students often don't have enough opportunities for social learning and development of soft skills. Flipped classrooms can provide social interaction through approaches such as lightweight teams [23], where students collaborate during class in low-stakes peer learning. These teams scaffold positive interdependence [17] by removing high-stakes assignments that heavily impact student's grades.

Given the proliferation of online courses, and MOOCs in particular, it is important to consider whether successful face-to-face pedagogical strategies can be reappropriated for distributed, online contexts. Specifically, we are interested in whether a low-stakes model could provide similar learning benefits when team members collaborate remotely.

This paper presents results from a study that analyzed the efficacy of low-stakes distributed teams. We examined whether low-stakes teams that communicate through Google Hangouts can provide educational benefits, in terms of both engagement and learning outcomes, compared to students who are learning via video in a co-located setting or individually. Results suggest that co-located teams have the highest learning gains, but there are no significant differences between distributed teams and individual work. We discuss implications of these results for practice and future research.
\end{abstract}

\section{Categories and Subject Descriptors}

K.3.2 [Computer Science Education]

\section{General Terms}

Education

\section{Keywords}

lightweight teams, distance learning, cooperative learning, team-based learning, computer science education

Permission to make digital or hard copies of all or part of this work for personal or classroom use is granted without fee provided that copies are not made or distributed for profit or commercial advantage and that copies bear this notice and the full citation on the first page. Copyrights for components of this work owned by others than ACM must be honored. Abstracting with credit is permitted. To copy otherwise, or republish, to post on servers or to redistribute to lists, requires prior specific permission and/or a fee. Request permissions from Permissions@ acm.org.

ICER'15, August 9-13, 2015, Omaha, Nebraska, USA.

(C) 2015 ACM. ISBN 978-1-4503-3630-7/15/08 ...\$15.00.

DOI: http://dx.doi.org/10.1145/2787622.2787727.

\section{INTRODUCTION}

The ubiquity of technology in society generates a constant demand for students with computer science skills and consequently enrollment in traditional four-year CS programs is high. Meanwhile, adoption of, and enrollment in MOOC platforms is also significant for CS, as shown by Stanford's 2011 Machine learning course that attracted 104,000 people [41]. Students in CS classes have varying levels of preparedness and diverse backgrounds. In both traditional college classes and MOOCs, the discipline of CS has focused almost exclusively on teaching skills and theory, at the expense of fostering social interaction, peer learning, and soft skills such as teamwork. This lack of soft skills may not only harm graduates when they enter the workforce, but it also means that the learning environments themselves are not optimized to take advantage of the benefits associated with social learning [35, 6, 40].

Fostering social interaction, teamwork, and peer learning in face-to-face courses often involves converting to flipped classrooms, where students consume video lectures online and then engage in active learning in the classroom [36, 37, 19, 22, 11]. Active learning and peer learning provide direct face-to-face interaction with peers and educators and create a much more social and engaging environment. When wellstructured, these cooperative learning opportunities have the potential to allow students to leverage their diverse backgrounds and experiences to provide new perspectives.

For online learning, social interaction is limited and happens primarily through discussion boards [4]. Anchored collaboration $[14,9,33]$ embeds these discussions into the media to which they correspond and provides support when students first interact with materials online. Asynchronous peer learning is amenable to conflicting schedules and, like face-to-face peer learning, discussion forums can make the diverse backgrounds of CS students an asset rather than a challenge. However, asynchronous peer learning doesn't provide rich face-to-face interaction and may not alleviate feelings of isolation. This isn't to imply a dichotomy where either asynchronous or synchronous learning is better; each have strengths, weaknesses, and use cases [16].

Both flipped classrooms and online classes rely on selfregulated learning without providing support or scaffolding for students who may not possess those essential metacognitive skills. Successful self-regulated learning requires four levels, which begin with observation and imitation and move to self-control and finally self-regulation [32]. However, in online learning and the out-of-class portion of flipped 
classes, students can't observe how other students interact with material, and therefore can't observe and imitate them.

We hypothesize that synchronous peer interaction, when students first interact with materials, encourages social interaction and allows students to observe and imitate each other's self-regulatory skills. For example, students may engage in collaborative problem decomposition in real-time. In this paper, we extend a pedagogical approach, Lightweight Teams [23], that has been shown to support students socially, mitigate stress associated with teamwork, and scaffold teamwork and communication skills. We test a similar low-stakes teams model that borrows the ideas of positive interdependence and stress-free teamwork but moves them to an online, distributed context where face-to-face communication is mediated by videoconferencing technology.

The contribution of this paper is to evaluate the efficacy of and the challenges associated with online, low-stakes, synchronous, team-based learning. In this work, we conduct a comparative study of co-located teams, distributed teams, and individual learning. The results from our comparative study corroborate previous team-based learning research and discussion-based peer learning research.

\section{BACKGROUND}

Our work builds on these topics in these ways:

- Collaborative Learning: provides a theoretical basis for the efficacy of Lightweight Teams.

- Flipped Classrooms and MOOCs: motivates our work and informs how our work could be applied.

- Distributed Teams and Online Discussion: prior work that investigates peer learning in an online context.

- Lightweight Teams: focus of our comparative study.

\subsection{Collaborative Learning}

It has been widely argued that students learn best when they actively collaborate on course work in small groups both inside and outside the classroom [35]. Lave et al.'s work on cultivating communities of learners [24] supports this notion, providing evidence that students become more engaged in their activities when they are part of such groups. The social context of learning allows the learners to engage in cognitive processes that would be otherwise unavailable if they were working individually [29, 39]. Specifically, Vygotsky argued that knowledge is constructed through social interaction and interactive talk is a tool mediating the advancement of thinking [39]. From this sociocultural perspective, when an individual interacts with other individuals in a social environment, internal processes occur that support learning. Most tasks, and learning in particular, require social interactions for ideas to develop [6, 40].

Cooperative learning, like collaborative learning, has been found to improve student achievement and self-esteem, and to develop positive race relationships [34]. Peer interaction is also important for scaffolding students' problem-solving processes [12]. In their meta-analysis on effects of small group learning, Springer et al. found that small groups have a positive impact on student outcomes and also increase persistence in STEM courses and programs [35]. Given the push to increase retention rates in MOOCs, synchronous small group learning offers a promising approach to keep students more engaged while learning online.

\subsection{Flipped Classrooms}

Group work is a common feature in flipped or inverted classrooms, where content delivery occurs outside of the classroom, and in-class time is devoted to active learning activities [36, 37, 19, 22, 11]. The active learning that occurs during class allows students to interact with peers and receive guidance from an instructor. To make this happen, students consume content as homework, so that they are ready to actively interact with content in class.

Post-secondary institutions are increasingly using the flipped class approach for large classes [22, 11, 1]. Large flipped classrooms can be intimidating for students and it can be a challenge to get the students to interact with each other. Dividing the students into smaller groups to collaborate on active learning activities during class time is proving to be a successful approach for increasing student engagement [31] and learning outcomes [15]. The flipped class approach to teaching opens up opportunities for student collaboration and peer learning, given that belonging to a group or a team can have significant positive benefits for individual courses and sustained success in college [2].

\subsection{MOOCs}

The recent popularity of MOOCs, especially in computing, as a mechanism to offer courses and in some cases, certificates, at no cost to students is changing how people think about higher education. This disruption is cause for both excitement and scrutiny [25]. Researchers who work in academia are particularly intrigued by this disruption that occurs so close to home, and much effort is now being targeted towards studying and understanding how MOOCs work and how effective they are at actually educating people $[10,30,3]$. In particular, it is important to pay attention to the differing communication patterns that emerge in the use of computer-mediated communication, since in the case of MOOCs, face-to-face communication is replaced by digital channels [18, 38]. Consequently, empirical studies are necessary to determine whether and how face-to-face pedagogical techniques translate to these partially or fully online environments.

\subsection{Distributed Teams and Online Discussion}

To support students working outside of class time or in completely online settings, such as MOOCs, it is important to understand how distance affects teamwork and cohesion. There are known difficulties associated with distributed teamwork and collaborating remotely. Specifically challenging for short-term ad-hoc teams, it is important to establish trust and common ground [26].

Despite these challenges, the benefits of peer learning and global perspectives have encouraged a variety of research around global virtual teams. Recently, Coursera introduced Talkabout[21], which provides students with an online, video-based chatroom for discussing course content. Talkabout enables globally diverse discussions by placing students from different countries into the same chatroom. A similar experiment used Mechanical Turk workers to participate in a synchronous text-based discussion forum [5] where participants answered questions independently, then discussed a short explanation, and finally re-answered the question independently. Coetzee et al. [5] have shown that discussing a question leads to better answers but it isn't clear whether this learning transfers to similar questions. 


\subsection{Lightweight Teams}

In our previous work [23], we defined Lightweight Teams as teams of students who work together in a course but where the work done as a team has minimal direct impact on an individual student's final grade. The idea is to get students talking to each other and engaging around the material in a social way, without forcing the students to work together on large, high-stakes, term-length projects. This means that students get the benefits of social learning, without the stress associated with high-stakes group work. Working with the same team throughout the length of the course gives the students a chance to get to know each other, become comfortable, and learn from one another.

Our lightweight teams were generated randomly and assigned seating, so team members always sat next to each other. Students never had to choose where to sit, who to sit with, or even wonder if someone would sit with them. We used a modified peer instruction method [7] to conduct the clicker quizzes ${ }^{1}$. Students discussed the question and answers with their teams before answering, but they were still able to answer individually without forming consensus. All of the clicker quizzes only accounted for $10 \%$ of each student's grade. The other peer learning activities included paper problem solving activities, where students were asked to write pseudocode, solve Parson's Problems [8], or create UML class diagrams.

Lightweight teams were used in several large introductory courses, where students worked in small groups during class time. In those classes, a marked change occurred and classrooms became a more friendly, social environment; quite unlike the environment typically associated with large introductory computing lectures. Compared to sections that didn't employ lightweight teams, students from lightweight team sections performed better; although this isn't a fair comparison because the two sections were taught in different languages ( $\mathrm{C}++$ and Java). Most importantly, a high percentage of students students indicated that they each made at least 5 new friends. Based on this success, lightweight teams seem to have particular promise as a pedagogical design pattern [13]. However, it is not known how well this design pattern transfers to a distributed online context.

\section{INTRODUCING THE DISTRIBUTED LOW-STAKES TEAM}

We take inspiration from previous distributed peer learning research, such as the synchronicity of Talkabout and the peer-instruction aspect of Coetzee's Study. We investigated the possibility of extending lightweight teams to an online environment where group members are distributed. In faceto-face (co-located) lightweight teams, students sit together in assigned seating to enable continuous interaction. Outside of class, we envision students using technology, such as Google Hangouts or Skype, to work together synchronously at pre-arranged times. In this experiment, we study lowstakes teams which borrow the low-stress, active learning and positive interdependence aspects of lightweight teams. However the team we study are different from lightweight teams in longevity, in that low-stakes teams meet once and do not have a chance to bond over time.

\footnotetext{
$\overline{{ }^{1} \text { http://www.turningtechnologies.com/higher-education }}$
}

\section{COMPARATIVE STUDY OF LEARNING IN LOW-STAKES TEAMS}

This research investigates whether the learning benefits observed from using lightweight teams in a face-to-face setting transfer to an online setting where participants are not co-located. To answer this question, we designed an empirical experiment that presented participants with new material and then asked questions to measure their learning of that material. Participants were split across three different conditions: learning individually, learning as a co-located group, and learning as a distributed group.

\subsection{Task and Procedure}

The task given to our participants was to learn about using semi-automatic espresso machines. We chose this task because we expected that most participants would be unfamiliar with it, and it is a task that has significant enough complexity to create difficult questions, but low enough complexity that basics can be learned in about 15 minutes. The general procedure of the study was as follows:

Pre-test: Take a web-based test (demographics, attitude and 14 knowledge assessment questions). (10 minutes)

Videos: Watch a series of short YouTube videos about espresso machine operations. (10 minutes)

Learning Quiz: Complete peer-instruction quiz about making espresso. Group conditions discuss questions, but individuals answer them alone. (5-20 minutes)

Post-test: Take a web-based post-test (14 knowledge assessment questions + preferences $)$. (10 minutes)

Watching videos replicates the standard information delivery mechanism for both MOOCs and flipped classrooms. In our experiment, participants were free to watch videos repeatedly, to adjust the video playback speed, to pause and replay parts of the videos, and to skip parts of the videos. The learning quiz was the active learning component, used by lightweight teams in flipped classes, based on the peer instruction methodology [7]. The learning quiz is not for assessment, but rather helps students engage with the material and learn it more deeply. In practice, the learning quiz could be replaced by a different learning task such as paper-prototyping, pair programming, or requirement elicitation. We chose to use the learning quiz because it is the learning task that was used by our lightweight teams. Research has shown that the act of recalling information serves to increase the retention of that information [27]. In addition, synthesizing information to answer complex questions moves learning from simple remembering in Bloom's revised taxonomy to understanding, applying, and analyzing [20].

In the two group conditions, the learning quiz that happens after watching the videos is a form of collaborative test taking [28]. We expected that participants in the group conditions would do better on these quizzes since they are able to talk about the question before answering, based on the 'wisdom of the crowd' effect. We also expected there to be a fair amount of consistency on these answers within each group, although each participant answered the questions individually and the group didn't need to reach consensus. For participants in the individual condition, this learning quiz was completed alone, and so the scores reflected only what the individual understood from watching the videos. 
Table 1: Pre- and Post-Test Knowledge Assessment Questions

\begin{tabular}{|l|c|}
\hline Question (asked as multiple choice) & Bloom's Category \\
\hline \hline Describe what it means when your espresso shot is over-extracted. & Comprehension \\
\hline Choose the boiler type that is most common in coffee shops. & Knowledge \\
\hline Select the ideal order of steps to make a cappuccino using a single-boiler espresso machine & Analysis \\
\hline Select the correct amount of time a it should take for a shot to pour & Knowledge \\
\hline Define a portafilter & Comprehension \\
\hline Select the ideal temperature for espresso & Knowledge \\
\hline Indicate a cause of a shot pouring too slowly & Evaluation \\
\hline Predict what can happen if your shot pours too slowly & Application \\
\hline Describe the taste of a shot that pours too slowly & Analysis \\
\hline Describe the taste of a shot that pours too fast & Analysis \\
\hline Identify the most likely cause of a damp puck & Knowledge \\
\hline Describe how the shot will pour during the first few seconds of pulling a shot & Comprehension \\
\hline Describe how you might know that your shot is finished & Comprehension \\
\hline Choose the benefit typically associated with burr grinders & Knowledge \\
\hline
\end{tabular}

The pre-test and post-test activities are representative of the types of questions that might be asked on a test in either a flipped course or a MOOC. While it is not standard for students in flipped classes or MOOCs to take a pre-test before being presented with new information about a concept, this was necessary for us to measure learning gains and to control for participants who might already have some knowledge and experience with the subject matter.

\subsection{Study Methodology}

We used a between groups design with three conditions:

Individual (I) : Participant did all parts of the study in an experiment room by themselves (control condition).

Group Co-located (GC): Participants did the study in a co-located group in an experiment room, working with the participants in their group on all parts of the study except the initial and final learning outcomes tests.

Group Distributed (GD): Participants did the study in individual experiment rooms. They worked individually except during the learning quiz when they discussed questions via Google Hangouts.

\subsection{Study Environment}

The participants in the co-located group completed the study in a conference room on our campus. The students in the distributed group completed the study from separate conference rooms and offices on our campus. The individual participants completed the study from either an office on campus (14 participants) or from their home (3 participants). Participants used their own equipment (laptop to login to the study, complete the tests and watch the videos); however, we also provided laptops if students were unable to join Google Hangouts. For participants in the distributed condition, this meant that they were using their own computers, microphones, web cams and speakers or headphones to connect to the Google Hangout to participate in the study with their assigned group. While this lead to technical glitches, we felt that this was closest to the real-world situation in which MOOC and distance learning students have to cope with using their own technology to participate.

\subsection{Research Question and Hypotheses}

Our general research question was whether low-stakes teams could provide educational benefit, in terms of learning outcomes, to students who are learning at a distance. We had the following hypotheses:

H1. Learning outcomes will be highest for the students in the co-located group.

H2. Learning outcomes will be higher for students in the distributed group than for students working alone.

H3. Students in both group conditions will perceive that group interactions improved their learning.

We expected that the best learning outcomes would occur for co-located participants, as current technology for distributed discussion limits interaction in the following ways:

- Videoconferencing technology (VCT) allows people to multitask inconspicuously.

- Gesturing and eye contact aren't as natural in VCT; it may be harder to negotiate who speaks.

- Lags and disconnections in VCT can cause conversations to last longer and fatigue attendees.

- Lags in VCT degrade the quality of the conversation.

- People working remotely do not have the freedom to sketch on paper to share visual ideas as easily.

Despite these technological limitations, we expected that participants in distributed teams would exhibit higher learning outcomes than participants in the individual condition, who had no-one to discuss the material with.

\subsection{Data Collection}

We created a web-app that allowed participants to take the tests and watch the videos in the same window. The only exception, where students were required to leave the webapp, was when distributed team members discussed questions in the Google Hangout. The investigator was present during the Google Hangout session to ensure that conversation stopped after the learning quiz when participants were required to work independently again. The learning quiz was 


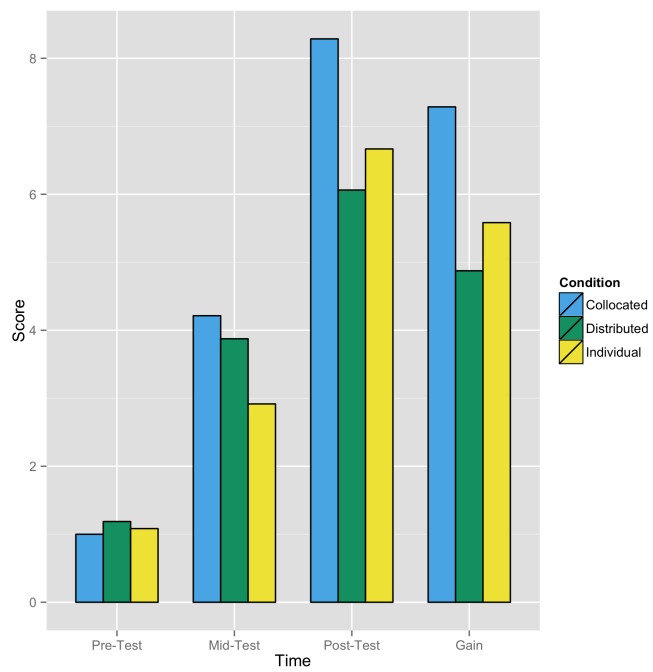

Figure 1: Average scores for the three tests, along with average gain, broken down by condition.

audio recorded for both face-to-face discussions and Google Hangout discussions. The web-app monitored the participants' interactions and then updated a server every 15 seconds with results for each section. This prevented participants from losing work and from artificially resetting the timer. All of the questions were optional and some participants did not answer all of the preference questions; however, all participants answered all scored questions.

\subsection{Quantitative Data}

The quantitative data included the pre- and post-test scores, and included the learning quiz scores. The preand post-tests contained 14 identical knowledge assessment questions; shown in Table 1 with their Bloom's taxonomy categorization. The pre-test primed participants on what they should attempt to learn from the videos. Bloom's taxonomy was used to ensure that questions were varied and didn't simply target one type of learning. The difference between pre- and post-scores on these 14 questions represents the learning gain. The demographic information collected included gender, age range, and race. We asked questions on the pre- and post-test to ascertain any affect that our experiment had on participants' preferences; shown in Table 5. Finally, we asked students how they perceived their experience, as summarized in Table 6 .

\subsection{Qualitative Data}

For participants in condition GC, we audio-recorded the session. For participants in the GD condition, we audiorecorded their Google Hangout session. These recordings were used to qualitatively analyze the interactions and also as an archive of what happened during the experiment.

\subsection{Participants}

We recruited participants from the student population at UNC Charlotte. In total, 53 participants (21 female) participated, and were divided among the three conditions based upon availability to participate during a group slot versus an individual slot. Participants were mainly majoring in com-

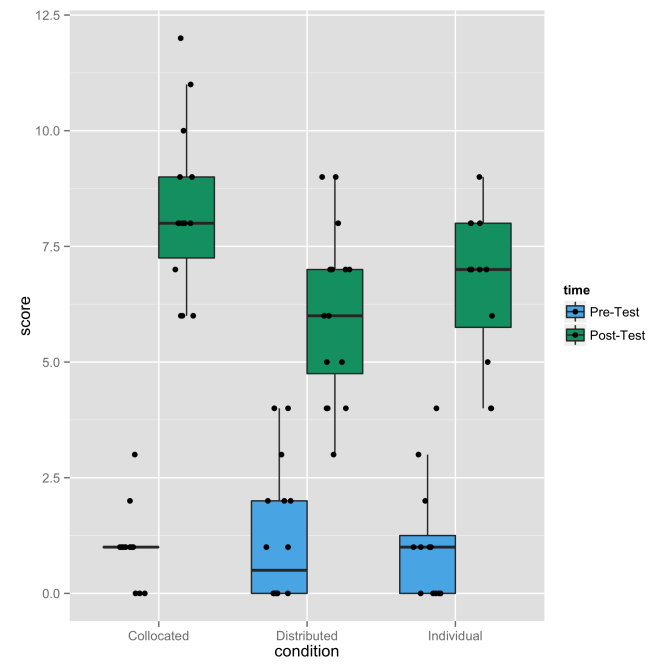

Figure 2: Box plots of pre- and post-test scores grouped by condition. Points are jittered to avoid over-plotting.

puter science or software information systems; although, a few were from Engineering and Bioinformatics. We had four distributed groups (three with five participants each, one with three participants), four co-located groups (two with five participants each, two with four participants), and 17 participants in the individual condition. Participants were compensated for their time (typically 40-60 minutes) with a gift card for a local coffee shop.

\subsection{Statistical Analysis}

The differences in learning gains between the three conditions were analyzed using a linear mixed effect model (LME) fitted by restricted maximum likelihood. In our model, posttest score was the dependent variable, condition (co-located, distributed, and individual) was a between subjects fixed effect and pre-test was our within subjects term. Participants were treated as random effects. For statistically significant observations $(\mathrm{p}<.05)$, we performed multiple comparison of means using Tukey contrasts applied to the LME models, which are reported using normally distributed $\mathrm{Z}$ values. When performing multiple comparisons, p-values were corrected using single-step method to control for family-wise error rates. We performed this same analysis to analyze scores on the active learning quiz, with learning quiz scores replacing post-test scores as the dependent variable in our model. For each LME analysis, normality and homoscedasticity of standardized residuals were checked using residualto-predicted plots and Q-Q plots (quantile-quantile).

To corroborate our findings, we performed a simple ANOVA of gain scores (post - pre) as the dependent variable and condition (GC, GD, I) as the independent variable. This approach doesn't account for repeated measures and won't have as much statistical power as our LME, which accounts for repeated measures. ANOVA of gain also assumes equal pre-test scores. For these reasons, we chose to use LME and only report the ANOVA of gain, shown in Table 3, for reference. The results are very similar to those found in our LME models, shown in Table 2. In the following sections, we will only report results from the LME approach. 
For preferences, we used a simple ANOVA to compare groups where their preference is the dependent variable. For each ANOVA, we used Levene's Test to ensure homogeneity of variances and this assumption was not violated. For preferences, we observed minor departures from normality. When significant, we performed post-hoc analysis using Tukey HSD test.

After the experiment concluded, we removed 3 participants (two from GC, one from I) because their language barrier was too great, as evidenced by the use of a webtranslator during the experiment to answer questions. After removing these 3 participants, but before conducting our analysis, we investigated the distributions of pre-test scores using histograms. We identified outliers and uneven mean values between groups for pre-test scores. To create a fair comparison, we removed all data for participants with pretests greater than or equal to 5 . The score was chosen because it represents 2 standard deviations from the global pre-score mean. After removing these participants our histograms indicated relatively normal distributions with positive skew and leptokurtosis because many scores existed at one and zero. Similarly, the pre-test scores were comparable between groups: $\mathrm{GC}(\mathrm{M}=1.0, \mathrm{SD}=0.8), \mathrm{GD}(\mathrm{M}=1.2$, $\mathrm{SD}=1.4)$ and $\mathrm{I}(\mathrm{M}=1.1, \mathrm{SD}=1.3)$. Thus our analysis only includes 16 participants in condition GD, 14 participants in condition GC, and 12 participants in condition I. While this reduced our statistical power, we believe it represents a fairer comparison between groups.

Table 2: LME Learning Gain: Multiple Comparisons of Means Using Tukey Contrasts (single-step corrected: significant if $<0.05$ )

\begin{tabular}{|l|c|c|c|}
\hline Condition & Estimate & Error & $\operatorname{Pr}(>|\mathrm{z}|)$ \\
\hline Distributed - Co-located & -2.32 & 0.61 & $<0.001$ \\
Individual - Co-located & -1.66 & 0.65 & 0.029 \\
Individual - Distributed & 0.65 & 0.63 & 0.550 \\
\hline
\end{tabular}

Table 3: Post-hoc Pairwise Analysis of Learning Gains in Between Condition by ANOVA to Corroborate Our LME Analysis of Gain.

\begin{tabular}{|l|c|c|}
\hline Conditions & Difference & Tukey p-value \\
\hline Distributed - Co-located & -2.41 & 0.001 \\
Individual - Co-located & -1.70 & 0.044 \\
Individual - Distributed & 0.71 & 0.539 \\
\hline
\end{tabular}

Table 4: LME for Learning Quiz: Multiple Comparisons of Means Using Tukey Contrasts (single-step corrected: significant if $<0.05$ )

\begin{tabular}{|l|c|c|c|}
\hline Condition & Estimate & Error & $\operatorname{Pr}(>|\mathrm{z}|)$ \\
\hline Distributed - Co-located & -0.37 & 0.41 & 0.633 \\
Individual - Co-located & -1.31 & 0.44 & 0.008 \\
Individual - Distributed & -0.94 & 0.43 & 0.068 \\
\hline
\end{tabular}

\subsection{Quantitative Results}

Figure 1 shows averages broken down by condition. The box plot in Figure 2 shows the pre-test and post-test score distributions by condition. What is interesting in interpreting these basic results are that the co-located and individual participants saw steady improvement from pre-test to learning quiz to post-test; suggesting that their understanding of espresso improved over time and with exposure to the material. However, for distributed teams, the act of discussing material led to improved scores on the learning quiz, but these did not translate to improved post-test scores.

Our findings for overall learning gains, summarized in Table 2 and corroborated in Table 3, show that co-located teams outperformed both individuals and distributed teams. These findings are statistically significant; however, we did not observe a statistically significant difference between distributed teams and individuals.

For the learning task, shown in Table 4, our results show statistically significant differences between all conditions except for the two team conditions.

These observations corroborate Coetzee's Mechanical Turk study [5] which showed that discussing questions leads to more correct answers; however, for distributed teams this understanding doesn't appear to have carried over to similar questions. This may suggest some aspect of social loafing or an artifact of 'wisdom of the crowd'. This suggests that longitudinal studies involving transfer questions are essential when evaluating distributed teams.

\subsubsection{Hypotheses}

We began this study with the following three hypotheses:

H1. Learning outcomes will be highest for the students in the co-located group.

H2. Learning outcomes will be higher for students in the distributed group than for students working alone.

H3. Students in both group conditions will perceive that group interactions improved their learning.

Our results support our first hypothesis. GC (co-located) participants outperformed GD (distributed) participants by an average of 2.32 questions answered correctly and outperformed I (individual) participants by 1.67 questions.

We did not obtain statistically significant results to support our second hypothesis. GD participants had similar learning gains to I participants. This result has several possible explanations: GD participants communicated using technology and encountered technical difficulties which were not present in the other two conditions. In our study, many students were using Google Hangouts for the first time. It is also possible that distributed teams have more trouble quickly developing a conversational rapport that is need for this type of learning.

We did not obtain evidence to support our third hypothesis. There was a statistically significant difference between groups for perceived learning (ANOVA $F=3.5, d f=2, p=$ 0.039); however, this difference was between the two group conditions (GC reporting .68 higher than GD on a five-point likert scale). Neither group condition was significantly different from the individual condition. There was also no statistically significant difference for perceived enjoyment. 
Table 5: Participant Preferences about Group Work, by Condition. Numbers are counts of participants expressing the preference. For some questions, participants didn't indicate a preference.

\begin{tabular}{|l|c|c|c||c|c|c||c|c|c||}
\hline Question Statement & I Pre & GD Pre & GC Pre & I Post & GD Post & GC Post & I Dif & GD Dif & GC Dif \\
\hline I work better in group projects & 9 & 7 & 10 & 10 & 10 & 9 & 1 & 3 & -1 \\
I work better alone on projects & 5 & 8 & 1 & 4 & 6 & 3 & -1 & -2 & 2 \\
\hline I learn better in groups & 8 & 7 & 6 & 6 & 12 & 10 & -2 & 5 & 4 \\
I learn better alone & 6 & 9 & 6 & 8 & 4 & 2 & 2 & -5 & -4 \\
\hline
\end{tabular}

Table 6: Participant Perception Questions (Likert Scale: 1=Highly Disagree and 5=Highly Agree).

\begin{tabular}{|l|c|c|c|}
\hline Question Statement & I Average & GD Average & GC Average \\
\hline I enjoyed learning about espresso machines. & 4.2 & 3.9 & 4.0 \\
I learned a lot during this experiment. & 4.0 & 3.8 & 4.4 \\
I would be able to use an espresso machine in real life. & 3.6 & 2.9 & 3.5 \\
\hline
\end{tabular}

\subsubsection{Preferences}

Table 5 shows participant preferences regarding learning and working in groups in general, and Table 6 shows their perceptions about their learning in this study. Differences were only significant for perceived learning (ANOVA $F=3.5, d f=2, p=0.039)$. Co-located participants were significantly more likely to express that they learned more about using an espresso machine than participants in the distributed condition $(\mathrm{p}=0.031)$. We also investigated any effect that the experiment had on participant's preferences by comparing differences between participant's pre-test to post-test preferences. We found no significant change in preference related to working in groups; however; we noticed changes in participants' preferences about learning in groups. We observed that typically participants preferred the condition to which they were assigned; likely a 'good participant' artifact.

\subsection{Qualitative Observations}

Our participants experienced many of the potential difficulties that students typically encounter during traditional courses and more frequently in online and MOOC-based courses. These difficulties include hardware inconsistencies, student language barriers, and scheduling problems.

Technical difficulties were prevalent for distributed teams, where participants used Google Hangouts. In this condition, two of the four groups had microphone issues which forced two members to use Google chat. Without prompting, team-mates read the muted participant's comments to the group and the participant with the "lesser" modality wasn't substantially limited in terms of their ability to contribute. These and other technical difficulties caused distributed teams to take longer to finish the experiment and typically the team took the whole hour. For individuals, the experiment was much faster, sometimes as short as 35 minutes. The distributed groups typically felt rushed because of the technical issues and didn't always fully watch all of the videos. This was in contrast to the co-located groups who all watched all of the videos, and the individuals who all watched all the videos (with one exception - one individual reported that they missed watching one of the videos).

We observed that participants with severe language barriers performed poorly, but their presence did not appear to negatively impact their team-members. In fact, because of the language barrier, these groups discussed each option in depth to help the person with the language barrier understand. Of the participants used in the analysis, we had numerous participants who spoke English as a second language: 7 in co-located, 5 in distributed and 4 in individual.

In general, multiple choice questions elicited more conversation than open-ended questions. There was also substantially more gesturing by participants in co-located teams. Finally, we were surprised that only one of the distributed participants muted his video, despite the fact that most participants didn't know each other.

Multiple perspectives provide students with information that may be missing from course materials. In one example from our study, participants were asked whether steaming milk or making espresso required higher temperatures. A video explained that espresso requires 200 degrees Fahrenheit but didn't mention steaming milk. Participants in group conditions often realized that water becomes steam at 212 degrees and therefore steaming milk requires higher temperatures; however, individual participants often did not.

The team dynamics for the two group conditions were interesting to observe. First, it was difficult to create ad hoc teams for this study, as participants had to be recruited to participate during the same time slot. There were instances of participants being late and one who didn't show up, causing one of our teams to have four instead of five participants. We generally noticed that in each team there were 'power participants' and 'weak participants'. The power participants tended to dominate and their answers, whether right or wrong, tended to be accepted by other group members. In some cases, we saw members 'appease' these power participants and pretend to agree with them, but then choose different answers. The weak participants tended to be ignored, even when their answers were correct. And, due to being ignored, these participants contributed less over time.

\section{DISCUSSION}

We investigated whether distributed low-stakes teams could provide the same educational benefits, in terms of both engagement and learning outcomes compared to co-located low-stakes teams and individuals. 
Our quantitative results show that learning gains are highest for co-located teams; supporting previous team-based learning research and lightweight teams. Results from the learning quiz corroborate findings from Coetzee's Mechanical Turk experiment [5] and suggest that presenting nonidentical post-test questions is necessary for discerning differences between students working in distributed teams. While our quantitative results were not able to support our hypotheses that distributed teams would outperform individuals, our qualitative observations tell a compelling story about the potential for distributed team-based learning.

Despite the language barriers and both hardware and software difficulties, the distributed teams did not perform significantly worse than individuals in our study. This is compelling because compared to individual learning, distributed teams offer many latent benefits, which are not easily measured - such as opportunities for networking and social interaction, an ability to observe and imitate other student's techniques for self regulated learning, and multiple perspectives to solve harder problems.

A few examples from our experiment lead us to believe that these latent benefits could be very important. We describe some of them, such as self-regulated learning, in the introduction and describe others, such as multiple perspectives and helping team-mates with technical difficulties, in the qualitative results section. We believe that these examples show a promising future for distributed teams. These examples show how participants can share their own personal perspective to clarify points of confusion. They demonstrate that teams collaborate to find ways to mitigate and even solve technical difficulties that arise. They suggest that students might feel more connected and create networks of like-minded students that may persist beyond the course.

Our specific design of distributed teams in this study can be considered one point in a large, multi-dimensional design space. If we picked a different point in this space, our distributed team might be larger in size, use Skype voice conference, and meet over successive time periods. Or we might create teams with complementary skills and have them engage using a virtual environment such as Second Life, where there are more affordances for social interaction. Thus, the fact that we did not see larger learning gains by distributed participants in our study does not mean that distributed team learning should be abandoned as a concept. There are many possibilities to explore in this design space, and we suspect that some future instantiation of distributed teams will show significant learning gains for participants.

\subsection{Limitations}

Having our participants use their own technology for this study is a double-edged sword. This choice lends real-world validity in that students use a variety of hardware and software to participate in online classes, and there are often technical glitches that limit or influence interaction. If we had provided hardware and software to our participants, we could have ensured that everyone had the same equipment and prevented the technical glitches that hampered the interaction of some of our participants. However, we also would have had to deal with some participants being unfamiliar with the systems we provided and that also could have hindered interaction. We opted for real-world validity and the accompanying technical glitches, but that choice does seem to have impacted some of the learning results.
In the flipped classroom deployment of lightweight teams, there is a strong temporal aspect: students get to know each other over the course of the semester and develop a relationship. Our study, consisting of a single session, does not replicate this dimension of lightweight teams. It is possible that the use of distributed low-stakes teams could lead to learning gains that are significant if teams are formed and allowed to work together over multiple sessions.

\section{CONCLUSIONS AND FUTURE WORK}

Our study showed that the use of co-located teams leads to higher learning gains. In our implementation of distributed teams, we didn't see similar benefits accrue for participants. We believe that the technical difficulties experienced by participants likely explains most of this. Our results support previous co-located team-based learning research and corroborate previous experiments that investigate distributed peer learning.

In future studies, we will provide a training/familiarity phase to ensure that participants are not encumbered by such technical problems. The fact that distributed teams performed similarly to individuals is promising because they additionally allow students to interact socially and develop soft-skills, such as self-regulation and teamwork. Thus, we believe that the use of lightweight teams holds promise outside of class where students may be partially or fully distributed. Clearly, environmental and temporal factors are important and many design decisions need to be investigated to better support distributed collaborative learning.

Because our co-located groups did so well, and there is already a grass-roots effort in many MOOCs to form colocated study groups, it may also be worth investigating ways to foster co-located learning outside of the classroom.

Further research is needed to understand how various factors impact learning outcomes and class engagement for distributed low-stakes teams. In particular, will the learning outcomes and engagement be improved by teams that work together as a group over time, or can teams be thrown together in an ad hoc way, meaning that each time a person joins a team, the team members are different? How does the size of the team impact the outcomes? We could even consider blended teams that are co-located during class and distributed outside of class-time.

And of course, more work is needed to investigate how specific modalities impact team work. Finally, there are reasons to believe that personality, gender and background all have significant impact on team dynamics. There is much work to do in looking at gender-matched teams; creating teams of students with complementary knowledge and skills and complementary personality traits; or creating teams that try to achieve demographic or other types of diversity. All of these factors could be amplified by interaction modalities that are less rich than face-to-face interaction.

\section{ACKNOWLEDGMENTS}

We would like to thank the members of the HCI lab at UNC Charlotte for piloting this study and providing feedback. This work was partially supported by the U.S. Department of Education under grant P200A130088. Any opinions, findings, conclusions, or recommendations expressed in this material are those of the authors and do not necessarily reflect those of the sponsor. 


\section{REFERENCES}

[1] K. Ash. Educators evaluate flipped classrooms. Education Week, 32(2):s6-s8, 2012.

[2] A. W. Astin. What matters in college, 1993.

[3] L. Breslow, D. E. Pritchard, J. DeBoer, G. S. Stump, A. D. Ho, and D. Seaton. Studying learning in the worldwide classroom: Research into edxï£is first MOOC. Research 8 Practice in Assessment, 8:13-25, 2013.

[4] D. Coetzee, A. Fox, M. A. Hearst, and B. Hartmann. Chatrooms in moocs: all talk and no action. In Proceedings of the first ACM conference on Learning@ scale conference, pages 127-136. ACM, 2014.

[5] D. Coetzee, S. Lim, A. Fox, B. Hartmann, and M. A. Hearst. Structuring interactions for large-scale synchronous peer learning. In Proceedings of the 18th ACM Conference on Computer Supported Cooperative Work \& Social Computing, CSCW '15. ACM, 2015.

[6] L. A. Coser. Men of ideas. Simon and Schuster, 1997.

[7] C. H. Crouch, J. Watkins, A. P. Fagen, and E. Mazur. Peer instruction: Engaging students one-on-one, all at once. Research-Based Reform of University Physics, 1(1):40-95, 2007.

[8] P. Denny, A. Luxton-Reilly, and B. Simon. Evaluating a new exam question: Parsons problems. In Proceedings of the Fourth International Workshop on Computing Education Research, ICER '08, pages 113-124, New York, NY, USA, 2008. ACM.

[9] B. Dorn, L. B. Schroeder, and A. Stankiewicz. Piloting trace: Exploring spatiotemporal anchored collaboration in asynchronous learning. In Proceedings of the 18th ACM Conference on Computer Supported Cooperative Work \& Social Computing, CSCW '15, pages 393-403, New York, NY, USA, 2015. ACM.

[10] D. Fisher. Warming up to MOOCs. Chronicle of Higher Education (Nov. 6, 2012), 2012.

[11] J. Foertsch, G. Moses, J. Strikwerda, and M. Litzkow. Reversing the lecture/homework paradigm using eteach $\AA$ web-based streaming video software. Journal of Engineering Education-Washington, 91(3):267-274, 2002.

[12] X. Ge and S. M. Land. Scaffolding studentsï£i problem-solving processes in an ill-structured task using question prompts and peer interactions. Educational Technology Research and Development, 51(1):21-38, 2003.

[13] P. Goodyear. Educational design and networked learning: Patterns, pattern languages and design practice. Australasian Journal of Educational Technology, 21(1):82-101, 2005.

[14] M. Guzdial and J. Turns. Effective discussion through a computer-mediated anchored forum. The journal of the learning sciences, 9(4):437-469, 2000.

[15] D. Horton, M. Craig, J. Campbell, P. Gries, and D. Zingaro. Comparing outcomes in inverted and traditional CS1. In Proceedings of the 2014 Conference on Innovation 85 Technology in Computer Science Education, ITiCSE '14, pages 261-266, New York, NY, USA, 2014. ACM.

[16] S. Hrastinski. Asynchronous and synchronous e-learning. Educause quarterly, 31(4):51-55, 2008.
[17] D. W. Johnson and R. T. Johnson. Making cooperative learning work. Theory into practice, 38(2):67-73, 1999.

[18] D. H. Jonassen and H. Kwon II. Communication patterns in computer mediated versus face-to-face group problem solving. Educational technology research and development, 49(1):35-51, 2001.

[19] B. Kelley, S. Miner, and F. Haggar. Technogogy and faculty development. Academic Exchange Quarterly, 16(1), 2012.

[20] D. R. Krathwohl. A revision of bloom's taxonomy: An overview. Theory into practice, 41(4):212-218, 2002.

[21] C. Kulkarni, J. Cambre, Y. Kotturi, M. S. Bernstein, and S. R. Klemmer. Talkabout: Making distance matter with small groups in massive classes. In Proceedings of the 18th ACM Conference on Computer Supported Cooperative Work \& Social Computing, CSCW '15, pages 1116-1128. ACM, 2015.

[22] M. Lage, G. Platt, and M. Treglia. Inverting the classroom: A gateway to creating an inclusive learning environment. The Journal of Economic Education, $31(1): 30-43,2000$

[23] C. Latulipe, N. B. Long, and C. E. Seminario. Structuring flipped classes with lightweight teams and gamification. In Proceedings of the 46th ACM Technical Symposium on Computer Science Education, pages 392-397. ACM, 2015.

[24] J. Lave and E. Wenger. Situated learning: Legitimate peripheral participation. Cambridge university press, 1991.

[25] J. Mackness, S. Mak, and R. Williams. The ideals and reality of participating in a MOOC. In Networked Learning Conference, pages 266-275. University of Lancaster, 2010.

[26] G. M. Olson and J. S. Olson. Distance matters. Human-computer interaction, 15(2):139-178, 2000.

[27] H. L. Roediger and J. D. Karpicke. Test-enhanced learning taking memory tests improves long-term retention. Psychological science, 17(3):249-255, 2006.

[28] A. Russo and S. H. Warren. Collaborative test taking. College Teaching, 47(1):18-20, 1999.

[29] G. Salomon, D. N. Perkins, and T. Globerson. Partners in cognition: Extending human intelligence with intelligent technologies. Educational researcher, 20(3):2-9, 1991.

[30] E. Schneider. Welcome to the MOOCspace: a proposed theory and taxonomy for massive open online courses. In AIED 2013 Workshops Proceedings Volume, page 2, 2013.

[31] N. M. Schullery, R. F. Reck, and S. E. Schullery. Toward solving the high enrollment, low engagement dilemma: A case study in introductory business. International Journal of Business, Humanities and Technology, 1(2):1-9, 2011.

[32] D. H. Schunk and B. J. Zimmerman. Social origins of self-regulatory competence. Educational psychologist, 32(4):195-208, 1997.

[33] V. Singh, C. Latulipe, E. Carroll, and D. Lottridge. The choreographer's notebook: a video annotation system for dancers and choreographers. In Proceedings 
of the 8th ACM conference on Creativity and cognition, pages 197-206. ACM, 2011.

[34] R. E. Slavin. Cooperative learning. Review of educational research, 50(2):315-342, 1980.

[35] L. Springer, M. E. Stanne, and S. S. Donovan. Effects of small-group learning on undergraduates in science, mathematics, engineering, and technology: A meta-analysis. Review of educational research, 69(1):21-51, 1999.

[36] J. Strayer. The effects of the classroom flip on the learning environment: a comparison of learning activity in a traditional classroom and a flip classroom that used an intelligent tutoring system. $\mathrm{PhD}$ thesis, The Ohio State University, 2007.

[37] J. Thornburg. If you build it, will they come?-flipping the classroom. Mining Key Pedagogical Approaches, page $79,2012$.
[38] D. Uribe, J. D. Klein, and H. Sullivan. The effect of computer-mediated collaborative learning on solving ill-defined problems. Educational Technology Research and Development, 51(1):5-19, 2003.

[39] L. S. Vygotsky. Mind and society: The development of higher mental processes, 1978.

[40] J. Weedman. Conversation and community: The potential of electronic conferences for creating intellectual proximity in distributed learning environments. Journal of the American Society for Information Science, 50(10):907-928, 1999.

[41] D. S. Weld, E. Adar, L. Chilton, R. Hoffmann, E. Horvitz, M. Koch, J. Landay, C. H. Lin, and M. Mausam. Personalized online education - a crowdsourcing challenge. In Workshops at the Twenty-Sixth AAAI Conference on Artificial Intelligence, pages 1-31, 2012. 